\title{
Large outbreaks of Chikungunya virus in Brazil reveal uncommon clinical features and fatalities
}

\author{
Luiz Tadeu Moraes Figueiredo ${ }^{[1]}$
}

[1]. Centro de Pesquisa em Virologia, Faculdade de Medicina de Ribeirão Preto, Universidade de São Paulo, Ribeirão Preto, SP, Brasil.

The emergence of arboviruses such as Chikungunya virus (CHIKV) in Brazil is a serious threat. CHIKV is an alphavirus (Togaviridae) that, in Africa, shifted from an enzootic maintenance cycle involving non-human primates to a humanmosquito-human cycle in which humans serve as amplification hosts and the anthropophilic mosquitoes Aedes aegypti and A. albopictus transmit the virus in urban settings ${ }^{1}$. Mutations of the original African strain improved the fitness of the virus in these anthropophilic vectors, allowing the spread of CHIKV worldwide $^{2}$. Cases of vertical transmission of CHIKV from mothers to their newborns have been reported ${ }^{3}$.

An Asian genotype of CHIKV was introduced to the Americas through the Caribbean in December 2013, and the first autochthonous Brazilian cases were reported in September 2014 in the northern state of Amapá. Interestingly, at the same time, another genotype of CHIKV, the East, Central and South African and Asian strain (ECSA), was found in Feira de Santana City, Bahia State (Northeast Brazil). CHIKV ECSA rapidly spread through other northeastern states ${ }^{4}$. In 2015, a total of 20,598 cases of Chikungunya fever were reported in Brazil. In 2016, more than 220,000 notifications were issued throughout the country; during the first semester of 2017 , outbreaks of CHIKV infection occurred in practically all Brazilian regions and were particularly severe in the States of Ceará and Minas Gerais, with more than 80,000 and about 20,000 cases, respectively ${ }^{4}$.

CHIKV was first recognized as a human pathogen in 1952 during an outbreak in Tanzania. The virus produces a selflimiting disease in most infected individuals. Chikungunya is an acute febrile illness with joint pain and a conspicuous rash. During the acute phase, the infection can produce high levels of viremia $\left[10^{9}\right.$ ribonucleic acid (RNA) copies $\left./ \mathrm{mL}\right]$. A humoral response based on neutralizing antibodies can be detected three days after the onset of illness; this is followed by a rapid virus clearance. The joint pain can be severe and accompanied by arthritis and, based on American studies, can become chronic in many patients ${ }^{5}$. In a subset of patients, mononuclear cells may

Corresponding author: Dr. Luiz Tadeu Moraes Figueiredo.

e-mail: 1tmfigue@fmrp.usp.br

Received 5 October 2017

Accepted 9 October 2017 become chronically infected with CHIKV. These cells were shown to trigger proinflammatory responses and osteoclastic activity in the bones showing joint destruction ${ }^{6}$. Chronic arthropathy as a sequel of most CHIKV infections is a serious public health problem. Large numbers of individuals who are unable to work due to chronic joint disease can cause major socioeconomic repercussions.

During large-scale CHIKV outbreaks such as those that occurred in Polynesia and Brazil, uncommon clinical features of Chikungunya were observed. Atypical and severe clinical forms of CHIKV infection have been seen in the elderly and in patients with underlying comorbidities such as hypertension, cardiorespiratory disabilities, and diabetes (among other chronic diseases). Unusual clinical presentations of CHIKV infections include hepatitis, myocarditis, hemorrhagic phenomena, central nervous system manifestations (meningitis/encephalitis), Guillain-Barré syndrome, retrobulbar neuritis, cardiac diseases (pericarditis/myocarditis), and congenital disease after maternalfetal transmission of the virus ${ }^{7,8}$.

Considering that patients can die during either, the acute or chronic phase of Chikungunya, due to lesions triggered by the virus itself or due to secondary complications of the infection, it is difficult to confirm deaths by CHIKV infection. Moreover, the concomitance of outbreaks of the dengue and Zika virus infection and the occurrence of a similar disease caused by Mayaro (an American alphavirus related to CHIKV) in Brazil complicate the identification of CHIKV infection as the cause of death. Despite these challenges, the Brazilian Ministry of Health recognized more than 200 deaths related to CHIKV infection in 2016 and more than 50 in $2017^{9}$.

CHIKV is widespread in Brazil, and other large outbreaks can be expected any time in cities infested by $A$. aegypti mosquitoes. In this context, it is crucial to analyze these outbreaks, including assessments of the most severe cases and deaths caused by CHIKV infection, as presented in an article in this issue of the Revista da Sociedade Brasileira de Medicina Tropical (RSBMT). Moreover, the physiopathology of the chronic joint disease caused by CHIKV should be studied to identify treatments that can mitigate this complication of the disease. Finally, vaccines that will likely be available shortly will be important tools for controlling the threat of CHIKV. 


\section{Conflicts of interest}

The author declares that there is no conflict of interest.

\section{REFERENCES}

1. Weaver SC, Charlier C, Vasilakis N, Lecuit M. Zika, Chikungunya, and other emerging vector-borne viral diseases. Annu Rev Med. 2018;69:1-14. doi: 10.1146/annurev-med-050715-105122

2. Figueiredo MLG, Figueiredo LTM. Emerging alphaviruses in the Americas: Chikungunya and Mayaro. Rev Soc Bras Med Trop. 2014;47(6):677-83.

3. Ramful D, Carbonnier M, Pasquet M, Bouhmani B, Ghazouani $\mathrm{J}$, Noormahomed T, et al. Mother-to-child transmission of Chikungunya virus infection. Pediatr Infect Dis J. 2007;26(9):811-5.

4. Nunes MRT, Faria NR, de Vasconcelos JM, Golding N, Kraemer MUG, de Oliveira LF, et al. Emergence and potential for spread of Chikungunya virus in Brazil. BMC Medicine. 2015;13:102.

5. Rodriguez-Morales AJ, Cardona-Ospina JA, Fernanda UrbanoGarzon S, Sebastian Hurtado-Zapata J. Prevalence of post-
Chikungunya infection chronic inflammatory arthritis: a systematic review and meta-analysis. Arthritis Care Res (Hoboken). 2016;68(12):1849-58.

6. Assunção-Miranda I, Cruz-Oliveira C, Da Poian AT. Molecular mechanisms involved in the pathogenesis of alphavirus-induced arthritis. Biomed Res Int. 2013;(2013): Article ID 973516, 11 pages. doi: $10.1155 / 2013 / 973516$

7. Economopoulou A, Dominguez M, Helynck B, Sissoko D, Wichmann O, Quenel P, et al. Atypical Chikungunya virus infections: clinical manifestations, mortality and risk factors for severe disease during the 2005-2006 outbreak on Réunion. Epidemiol Infect. 2009;137(4):534-41.

8. Torres JR, Leopoldo Códova G, Castro JS, Rodriguez L, Saravia V, Arvelaez J, et al. Chikungunya fever: atypical and lethal cases in the Western hemisphere. A Venezuelan experience. IDCases. 2014;2(1):6-10.

9. Cavalcanti LPG, Freitas ARR, Brasil P, Cunha RV. Surveillance of deaths caused by arboviruses in Brasil: from dengue to chikungunya. Mem Inst Oswaldo Cruz. 2017;112(8):583-5. 